\title{
ş \\ Optimized capture section for a muon accelerator front end
}

\author{
Hisham Kamal Sayed* and J. Scott Berg \\ Brookhaven National Laboratory, Upton, New York 11973, USA
}

(Received 24 April 2014; published 28 July 2014)

\begin{abstract}
In a muon accelerator complex, a target is bombarded by a multi-MW proton beam to produce pions, which decay into the muons which are thereafter bunched, cooled, and accelerated. The front end of the complex captures those pions, then manipulates their phase space, and that of the muons into which they decay, to maximize the number of muons within the acceptance of the downstream systems. The secondary pion beam produced at the target is captured by a high field target solenoid that tapers down to a constant field throughout the rest of the front end. In this study we enhance the useful muon flux by introducing a new design of the longitudinal profile of the solenoid field at, and downstream of, the target. We find that the useful muon flux exiting the front end is larger when the field at the target is higher, the distance over which the field tapers down is shorter, and the field at the end of the taper is higher. We describe how the solenoid field profile impacts the transverse and longitudinal phase space of the beam and thereby leads to these dependencies.
\end{abstract}

DOI: 10.1103/PhysRevSTAB.17.070102

PACS numbers: 41.75.Ak, 41.75.Cn, 41.85.-p

\section{INTRODUCTION}

Proposed muon accelerators, which include the muon colliders and neutrino factories, use intense muon beams to study the physics of elementary particles at both the intensity and energy frontiers. A muon collider collides bunches of opposite charge at energies as high as several $\mathrm{TeV}[1,2]$. A neutrino factory creates intense neutrino beams from the decay of muons which have been accelerated to energies ranging from $5 \mathrm{GeV}$ to a few tens of $\mathrm{GeV}[1,3]$.

Muons for these accelerator complexes are produced by bombarding a target with a high-power proton beam and allowing the resulting pions to decay into muons. The initial energy and transverse momentum spreads of these pions are extremely large. The front end of a muon accelerator should capture a large fraction of these pions and manipulate their phase space to achieve a smaller energy spread. It should maximize the number of muons that are accepted by the downstream cooling and/or acceleration systems, thereby increasing the luminosity of the muon collider or the neutrino flux of the neutrino factory.

The front end is a solenoid focused beam line that begins with a high field at the target, which is then tapered down to a lower field (the "final field"). The length over which the on-axis field tapers down to the final field value is called the taper length. As the on-axis solenoid field decreases, the transverse momenta of the captured pions are reduced,

\footnotetext{
*hsayed@bnl.gov

Published by the American Physical Society under the terms of the Creative Commons Attribution 3.0 License. Further distribution of this work must maintain attribution to the author $(s)$ and the published article's title, journal citation, and DOI.
}

while the beam size is increased. This process allows the capture of pions with relatively large transverse momenta and delivers them to a much lower field (lower cost) solenoid channel.

The main components of the muon front end are the target station, decay channel, buncher, phase rotator, and finally the ionization cooling channel. A schematic layout of the front end is shown in Fig. 1, and Fig. 2 is a diagram showing, for one particular example, the solenoid coils near the target along with the on-axis field they generate. A detailed description of the front end is given in Sec. II.

In this study, three parameters of the longitudinal profile of the solenoid field are varied: the field at the target, the final field, and the taper length. The impact of this field profile on the secondary pion beam phase space immediately after the target, the muon phase space at the end of the decay channel, and the overall performance of the muon accelerator front end is examined.

In our study, we first demonstrate that a higher field at the target improves the transverse emittance just after the target and increases the number of captured pions. Next, we show that while a longer taper leads to a smaller transverse emittance of the pion and muon beams, and therefore a larger number of transmitted muons at the end of the decay channel, a long taper causes a growth in the muon beam's longitudinal emittance. The longitudinal emittance growth arises from a nonlinear coupling between the transverse and longitudinal planes of motion (particles with large transverse angles have longer paths, hence taking longer times). The balance between the longitudinal emittance dilution of the long taper and the transverse emittance growth due to a very short taper yields an optimal solution for the taper length. Finally, we show that the capture efficiency of the front end is higher for larger values for the final field, due 


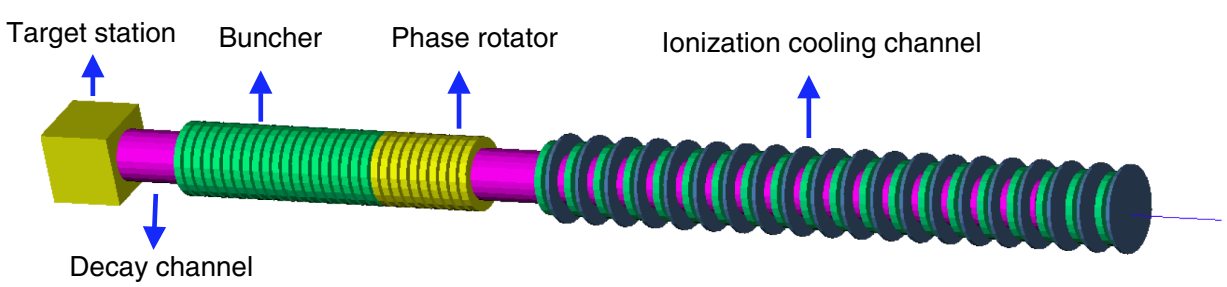

FIG. 1. Layout of the muon accelerator front end.

to the increased phase space acceptance of a solenoid for higher fields. We show that by optimally choosing the solenoid field profile, we can improve the performance of the front end over the existing design [5] by as much as $60 \%$.

A tapered solenoid field for secondary beam collection and transport was first introduced for positron sources [6]. The solenoid field is varied slowly with longitudinal position to maintain adiabatic invariance, where the single-particle action (the average of which is the emittance), when computed as though the field were not varying, is nearly constant as the particle travels down the beam line. Tapered solenoid fields were later adopted for capturing pions for a muon collider [7].

In [8] the capture solenoid field profile was examined, and it was found that the performance could be improved with a long adiabatic taper. The results of these studies were incorporated in a subsequent neutrino factory design [9], demonstrating an approximately $10 \%$ performance improvement over a longitudinal profile based on [7]. In all of these prior studies, performance was examined without consideration of the capture in the longitudinal phase space, which our study shows is essential.

We present the results of our study in Secs. III and IV. In Sec. III the impact of the target solenoid field profile on the phase space of the captured particles is studied. The impact of the peak field is presented in Sec. III A. In Sec. III B it is shown that a shorter taper increases the number of muons

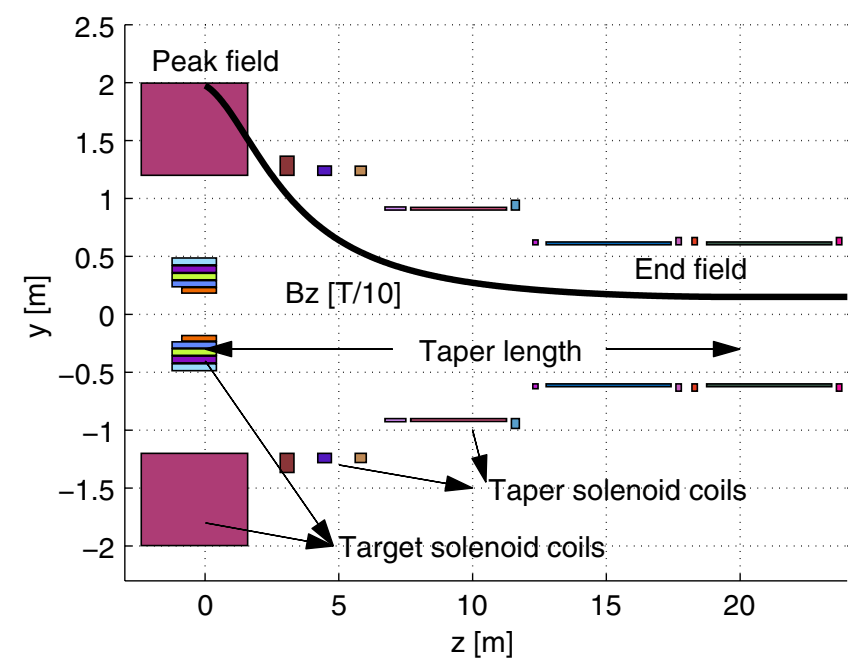

FIG. 2. Muon collider baseline target solenoid coils [4]. The on-axis solenoid field peaks at $20 \mathrm{~T}$ then tapers down to $1.5 \mathrm{~T}$. captured in the buncher and phase rotation because it reduces the effective longitudinal emittance growth due to the coupling of transverse motion into the longitudinal plane, and that the relative longitudinal emittance reduction from a shorter taper is much larger than the relative transverse emittance increase. It will be shown that it is optimal to have a much shorter taper than has been used in previous designs $[8,10,11]$. In Sec. III D we demonstrate that increasing the final field results in an increase in the number of captured muons. Finally, in Sec. IV we show that the advantage of a short taper continues to hold for proton driver bunches shorter than $20 \mathrm{~ns}$. The results of this study could in general be applied to any secondary beam collection channel.

\section{DESCRIPTION OF THE MUON ACCELERATOR FRONT END}

\section{A. Target system}

The current design of the target system for a muon collider or neutrino factory based on the machine designs developed by the International Design Study for a neutrino factory $[12,13]$ has a liquid mercury jet for a target within a $20 \mathrm{~T}$ solenoid field. A $4 \mathrm{MW}$ proton beam (about $3 \times 10^{15} 8 \mathrm{GeV}$ protons per second) intersects the mercury jet, producing low energy pions. The schematic of the target system and the on-axis field are shown in Figs. 2 and 3 $[13,14]$. The solenoid field is generated by concentric solenoids: a $5 \mathrm{~T}$ copper solenoid near the beam and a $15 \mathrm{~T}$ superconducting solenoid at a larger radius. The mercury jet and the proton beam are tilted with respect to the solenoid axis and each other. The portion of the mercury jet disrupted by the proton beam is replaced before the arrival of the following proton pulse. This configuration results in 0.8 pions at the end of the target per $8 \mathrm{GeV}$ proton.

Pion production was simulated with the MARS code [15]. The simulation was started with a proton beam with a Gaussian distribution at a location upstream of the peak of the target solenoid field. The momentum distribution of pions, kaons, and muons immediately after the target is shown in Fig. 4.

\section{B. Buncher, phase rotator, and ionization cooling channels}

After the solenoid field has tapered down to its final value, any remaining pions continue to decay into muons, producing a beam consisting almost entirely of muons by 


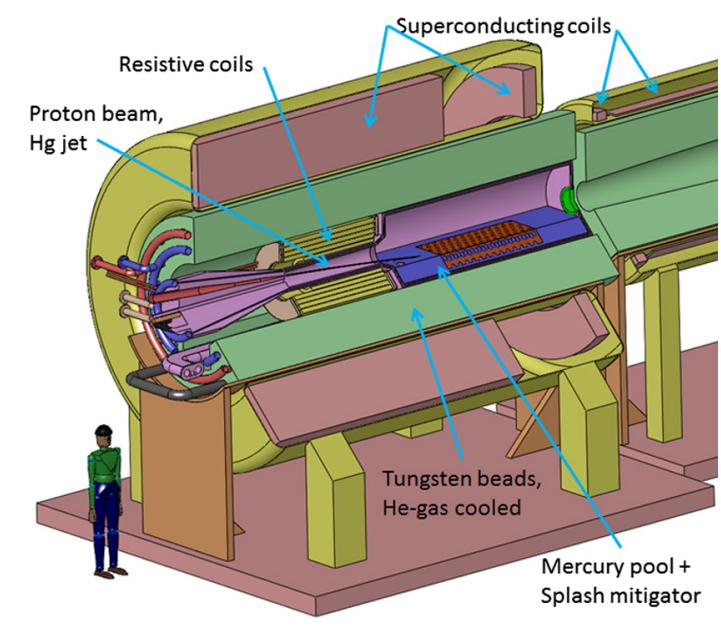

FIG. 3. Neutrino factory/muon collider target system.

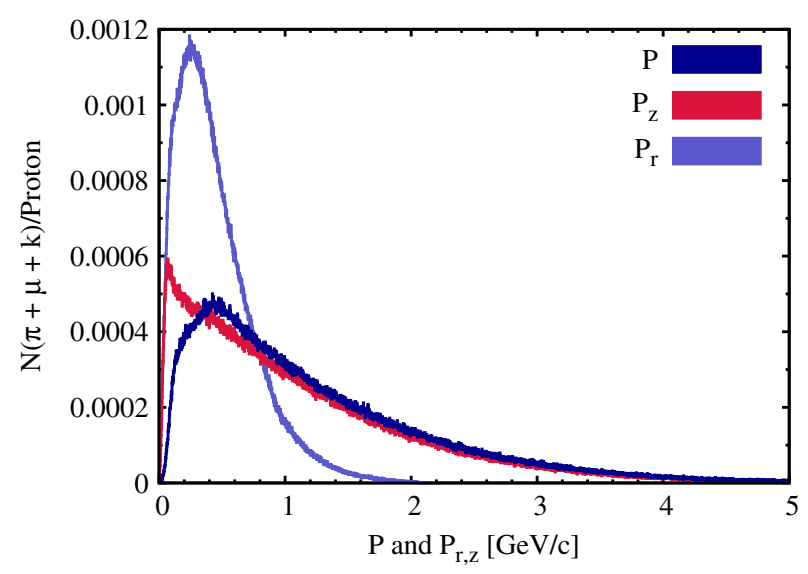

FIG. 4. Distribution in kinetic momentum of the sum of the charged pions, charged kaons, and muons, divided by the number of incident protons, at the end of the target. Distribution functions are plotted in total momentum (darker blue), longitudinal momentum (red), and transverse momentum (lighter blue). Transverse momentum is the magnitude of the projection of the momentum vector into the plane perpendicular to the solenoid axis.

the end of the decay channel. The muon bunch develops a correlation between time and energy as it propagates down the channel due to the variation of muon velocity with energy. Due to the broad energy spectrum of the pions produced at the target, the energy spread of the muons is much larger than the energy acceptance of the subsequent cooling and acceleration sections. The buncher and phase rotation sections transform this single bunch with a large energy spread into a bunch train with a small energy spread. We use the front end described in [5], except that we vary the field profile in the initial taper and the final field used through the buncher and phase rotation.

The solenoid field of $20 \mathrm{~T}$ at the target tapers down to reach its final value of $1.5 \mathrm{~T}$ at point $18.9 \mathrm{~m}$ from the end of the target. It then continues to maintain that field through the end of the phase rotation. It is this solenoid field profile that we will vary in this study. Once the field reaches its final value (which will be varied in our study), it continues with that value through the remainder of the decay channel, which extends to $79.6 \mathrm{~m}$ from the end of the target, and through the buncher and phase rotation sections.

The buncher [16] has a series of rf cavities with frequencies decreasing from 320 to $234 \mathrm{MHz}$ over a distance of $33 \mathrm{~m}$, while the rf gradients in the cavities increase from 0 to $10 \mathrm{MV} / \mathrm{m}$. The rf gradients increase adiabatically as the continuous distribution is transformed into a sequence of bunches. The $r f$ frequencies increase to keep the rf buckets centered on the bunches as the time between bunches increases due to their differing energies.

The buncher is followed by a $42 \mathrm{~m}$ phase rotation section that transforms the bunches, which all have different energies, until each bunch has nearly the same energy. It contains rf cavities which have frequencies decreasing down the channel from 230 to $202 \mathrm{MHz}$, and all having a gradient of $13 \mathrm{MV} / \mathrm{m}$. The cavity phases are set so that most bunches are at rf phases away from the zero crossing, such that the energies of all the bunches gradually become identical. The rf frequencies decrease so as to maintain these phases as the time between bunches increases due to their energy differences, and that decrease slows as the range of bunch energies is reduced.

The result is that muons with an initial momentum range of 80 to $500 \mathrm{MeV} / \mathrm{c}$ are captured into a train of 201.25 MHz bunches with an average momentum of $232 \mathrm{MeV} / c$ and a momentum spread of $10 \%$. The bunch train is approximately $60 \mathrm{~m}$ long, consisting of about 40 bunches of each charge. The front end captures muons of both charge signs, separated by $\pi$ in rf phase.

In the neutrino factory scenario, the phase rotator is followed by a transverse ionization cooling channel. This lattice is a sequence of identical $75 \mathrm{~cm}$ cells that focus with alternating solenoids producing a maximum field magnitude on axis of $2.8 \mathrm{~T}$. A $6 \mathrm{~m}$ section of solenoids and $\mathrm{rf}$ cavities, similar in structure to the cooling channel, is required to match between the constant solenoid field upstream and this alternating field. The cooling lattice contains $\mathrm{LiH}$ absorbers for ionization cooling and $\mathrm{rf}$ cavities that provide longitudinal focusing and restore the energy lost in the absorbers. The rf cavities have a frequency of $201.25 \mathrm{MHz}$ and an accelerating gradient of $16 \mathrm{MV} / \mathrm{m}$.

For some of our results we will use the cooling channel to measure the performance of our front end. We determine the number of muons within normalized acceptances of $30 \mathrm{~mm}$ transversely and $150 \mathrm{~mm}$ longitudinally at the end of each cooling cell (see [17] for details of the calculation), and take the maximum of that value. Early in the cooling channel, transverse emittance reduction increases the number of muons within the cuts. Later, the transverse emittance approaches an equilibrium, and then decays, 
stochastic processes, and longitudinal emittance growth from the cooling process create losses that exceed any increase in the transverse phase space density.

\section{EFFECT OF THE SOLENOID FIELD PROFILE ON PARTICLE CAPTURE EFFICIENCY}

To study the impact of the solenoid field profile on the performance of the front end, we first studied the effect of the target field on the beam just after the target. We then examined how the beam distribution and capture at the end of the decay channel is affected by the taper length. Next, we studied how the complete field profile affected the performance of the full system, including the buncher, phase rotation, and ionization cooling. We then describe how the final field affects the capture performance. Finally, we examined the effect of the proton bunch length on the performance of the system.

To simulate particle production at the target, we use the MARS15(2010) code [15]. We tracked through the subsequent decay channel, buncher, phase rotation, and cooling, using ICOOL [18].

\section{A. Target field and capture just after the target}

To understand the factors that govern the performance of the front end, we studied the effect of the field at the target on the particle distribution. We therefore vary the solenoid field at the target and examine the distribution just after the target. Figure 5 shows that the transverse emittance of beam decreases as the target field increases.

This can be understood in terms of the phase space dynamics in the target solenoid field. It is useful to work in

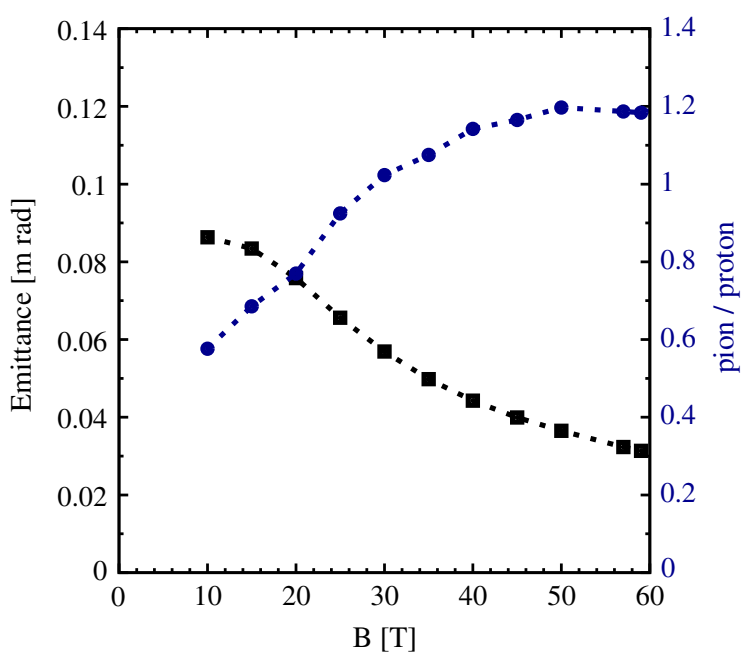

FIG. 5. The transverse emittance of secondary pions at the end of the target is stabilized by the target solenoid field as predicted by the analytic form. The number of captured pions at the end of the target increases with the focusing field strength reaching the maximum at $50 \mathrm{~T}$. a frame of reference (the Larmor frame) that rotates with twice the rotation period of the pions in the solenoid field [6]. In the approximation that the rms radius where the pions are produced is small compared to $2 \sigma_{p_{x}} /\left(e B_{z}\right)$, where $\sigma_{p_{x}}$ is the rms transverse momentum spread, $e$ the electron charge, and $B_{z}$ solenoid field, the normalized action $[19,20]$ in the Larmor frame for a single particle with a transverse momentum $p_{x}$ is given by $p_{x}^{2} /\left(e B_{z}\right)\left(I_{2}\right.$ in [6] is twice the action). This action will remain constant in a constant solenoid field, and will be nearly constant in a longitudinally varying solenoid field if the solenoid field varies slowly enough (if $\varepsilon$ from [6] is small enough; also see the discussion in the next subsection) [6]. Once the pions have gone far enough downstream to have undergone a number of rotation periods, we can safely assume that the distribution of particles in the angle variable will be relatively uniform due to two effects: first, that the finite length of the target means that to reach a given downstream position, different pions will have gone through a different number of betatron oscillations; and second, that particles with different energies undergo a different number of betatron oscillations over a fixed distance [Eqs. (16) and (15) in [6]]. The transverse normalized emittance is then just the average of the action [19] (this definition is consistent with [21]), which is $\sigma_{p_{x}}^{2} /\left(e B_{z}\right)$. Lichtenberg [22] applies a similar procedure for a different system to find a phase space distribution.

This emittance is intrinsic to the beam dynamics in the solenoid field. Earlier studies of pion capture for a muon accelerator $[7,8,11]$ generally required some reference to a solenoid aperture and a desired transverse momentum acceptance to define a desired target field. Our result, in contrast, makes it clear that a higher field at the solenoid by itself will lead to better performance (though this is also implicit in the calculations in [7], and consistent with their final result).

The emittance in Fig. 5 does not follow this inverse dependence on $B_{z}$ for smaller $B_{z}$ for two reasons: because particles reach large radii and are lost, and due to the lower betatron frequency at these fields (the distribution has not propagated for a sufficient number of betatron wavelengths for the transverse emittance to have reached its asymptotic value).

Fields above $20 \mathrm{~T}$ would be very challenging technologically. Not only is it difficult to make solenoids with such high fields, but the high radiation environment requires large amounts of shielding (out to a radius of $1.2 \mathrm{~m}$ for our example) inside the superconducting magnets. Therefore, for the remainder of this study, we will only consider target fields of 20 and $15 \mathrm{~T}$.

\section{B. Taper length and muon yield at the end of the decay channel}

Our field tapers down from the higher field $B_{i}$ at the target to a lower field $B_{f}$ at a distance $L_{t}$ downstream from 


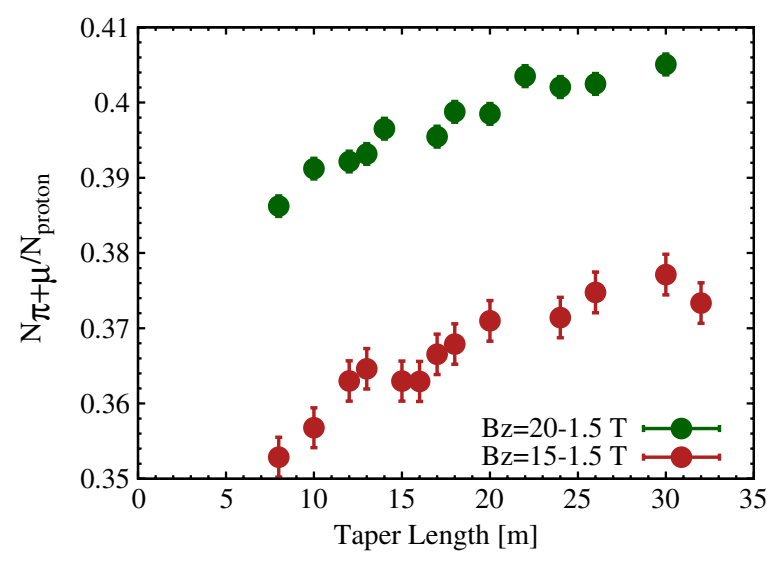

FIG. 6. Number of positively charged muons and pions counted $50 \mathrm{~m}$ downstream from the end of the target with a kinetic energy from 80 to $140 \mathrm{MeV}$ plotted vs taper length for target fields of 15 and $20 \mathrm{~T}$.

the target. The field on the solenoid axis at a position $z$ is given by

$$
\frac{B_{i} B_{f} L_{t}^{3}}{B_{i} z^{2}\left(3 L_{t}-2 z\right)+B_{f}\left(L_{t}-z\right)^{2}\left(2 z+L_{t}\right)} .
$$

The field is $B_{i}$ for $z<0$ and is $B_{f}$ for $z>L_{t}$. This is a specific case of the form used in $[8,11]$. Off the axis, the field is expanded as a power series in radius based on the longitudinal derivatives of the field on axis [23,24].

In order to match the $20 \mathrm{~T}$ peak solenoid field at the target with subsequent weak focusing channels with focusing field ranging from 1.5 to $3.5 \mathrm{~T}$ an adiabatic decrease of the field is adopted. The adiabaticity is valid in the regime where the length scale over which the magnetic field changes is large compared to the betatron wavelength, which for the solenoid channel means

$$
\frac{2 p}{q B^{2}} \frac{d B}{d z} \ll 1
$$

In order to study the impact of the taper length on the capture efficiency of the pions, particles were tracked for $50 \mathrm{~m}$ downstream from the end of the target. The number of muons and charged pions with a kinetic energy between 80 and $140 \mathrm{MeV}$ at that final position were counted. We studied fields of 15 and $20 \mathrm{~T}$ at the target, and taper lengths varying from 5 to $40 \mathrm{~m}$. The results are shown in Fig. 6. We see that the number of pions and muons in the decay channel is decreased by about $8 \%$ when we decrease the target field from its 20 to 15 T. Furthermore, a longer, more adiabatic taper increases the number of muons captured by the taper and decay channel.

\section{Solenoid taper length and beam phase space}

We simulated particle production and transport through the end of the decay channel using a field taper with a target field of $20 \mathrm{~T}$, a final field of $1.5 \mathrm{~T}$, and taper lengths of 4 and $40 \mathrm{~m}$. Figures 7 and 8 show the longitudinal phase space in these simulations. From these figures one can see that the bunch length is shorter and the longitudinal phase space density is higher for the shorter taper.

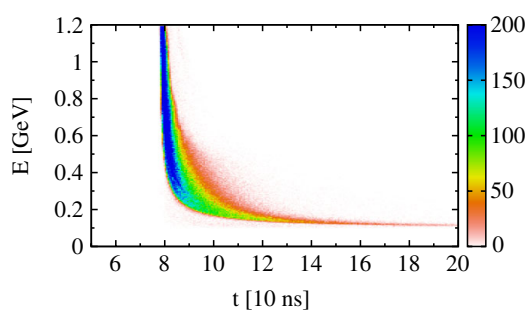

(a)

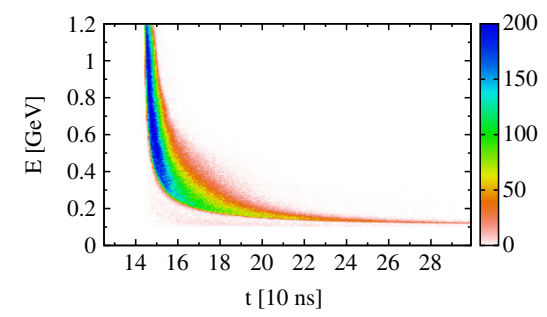

(b)

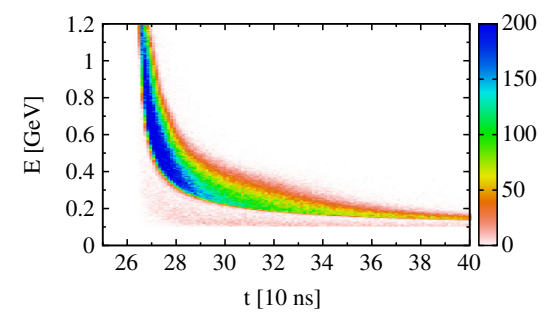

(c)

FIG. 7. Progression of the longitudinal phase space of pions exiting a $4 \mathrm{~m}$ long tapered solenoid. (a) $z=20 \mathrm{~m}$. (b) $z=40 \mathrm{~m}$. (c) $z=80 \mathrm{~m}$.

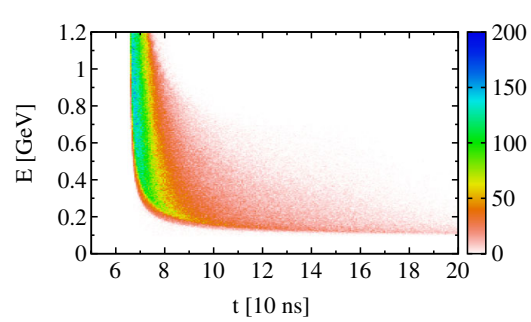

(a)

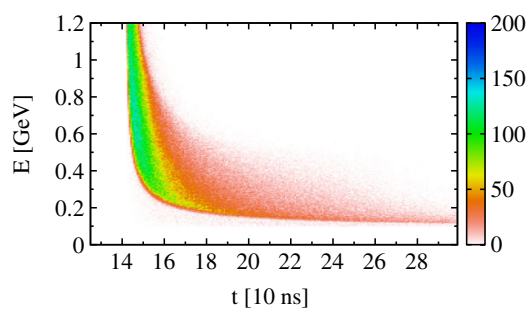

(b)

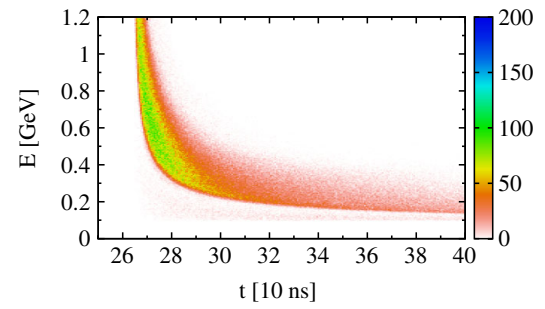

(c)

FIG. 8. Progression of the longitudinal phase space of pions inside [8(a)] and after exiting [Figs. 8(b) and 8(c)] from a $40 \mathrm{~m}$ long tapered solenoid. (a) $z=20 \mathrm{~m}$. (b) $z=40 \mathrm{~m}$. (c) $z=80 \mathrm{~m}$. 
We quantified this by simulating a larger number of different taper lengths and finding the rms time spread in the beam as a function of the taper length. To compute the rms time spread, we group the particles into 250 bins in total energy from $m_{\mu} c^{2}$ to $1 \mathrm{GeV}$. Within each bin, we find the rms time spread. We then average the time spread over the bins, weighting by the number of particles within each bin. The result is shown in Fig. 9. The bunch length increases significantly, and approximately linearly, with the solenoid taper length. This results in an increase in longitudinal emittance with increasing taper length, as shown in Fig. 10.

The reason for this increase in time spread is that particles with a given energy whose trajectories make larger angles with respect to the solenoid axis require a longer time to travel a given longitudinal distance than those which make smaller angles with respect to the axis. For a given transverse invariant action, higher magnetic

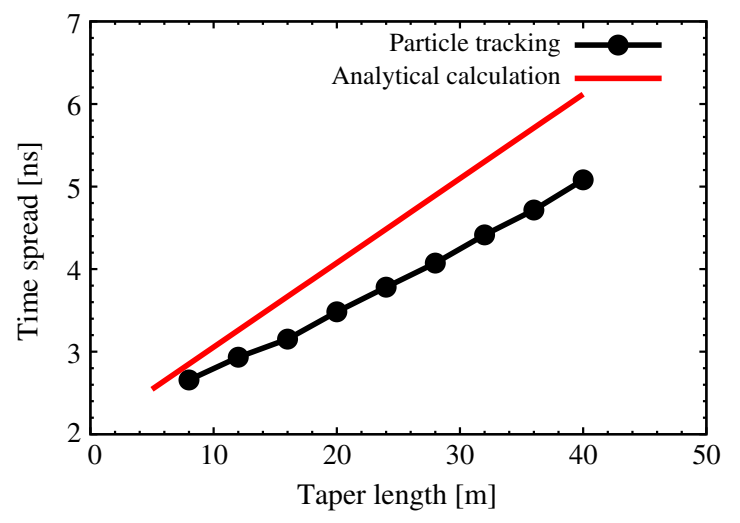

FIG. 9. The rms time spread of the muon beam at the end of decay channel as a function of the solenoid taper length. Simulations with our field number are shown with squares, the results of applying Eq. (3) to the distribution at the target are shown with a line.



FIG. 10. Transverse and longitudinal emittances of the muon beam at the end of decay channel as a function of the solenoid taper length, for the same simulations used in Fig. 9. fields will result in larger angles. As a result, we expect longer tapers which have more of their length at higher fields to cause a larger time spread in the beam at the end of the decay channel.

This can be quantified using the theory developed in [25]. We start with the approximations that the magnetic field is much smaller than what would be matched to the beam (we demonstrated this to be the case above), that the betatron oscillation wavelength is much smaller than the length scales over which the solenoid field varies, and that nonlinear effects beyond those described in [25] are negligible. We ignore effects arising from pion decays. Then the difference between the arrival time at a position $z$ of a particle with a nonzero transverse amplitude and that of a particle with zero transverse amplitude is given by

$$
\frac{\left(p_{x 0}^{2}+p_{y 0}^{2}\right) E}{2 p^{3} c^{2}} \int_{0}^{z} \frac{B_{z}(\bar{z})}{B_{z}(0)} d \bar{z}
$$

where $p_{x 0}$ and $p_{y 0}$ are the horizontal and vertical momenta of the particle at $z=0, p$ is its total momentum, $E$ is its energy, and $B_{z}(z)$ is the on-axis solenoid field. For a given target $(z=0)$ field and final field, this is a linear function of the taper length. To find the rms time spread arising from this (assuming all particles have the same time at $z=0$ ), one can simply find the standard deviation of the factor in front of the integral over the beam distribution at $z=0$.

As the taper becomes shorter and therefore less adiabatic, we expect some growth in transverse emittance. This is seen in Fig. 10. This explains the increased transmission shown in Fig. 6. Note that the reduction in the rms transverse emittance with a longer taper is much less than the increase in longitudinal emittance (Fig. 10).

\section{Performance with buncher, phase rotation, and cooling}

Only a subset of the muons at the end of the decay channel will be captured by the buncher and phase rotation, be subsequently cooled, and finally be within the acceptance of the acceleration system. We will determine the performance of the front end by finding the point in the cooling channel with the maximum number of muons falling within a $30 \mathrm{~mm}$ normalized transverse acceptance and a $150 \mathrm{~mm}$ normalized longitudinal acceptance (see [17] for details), and defining the performance to be that maximum number of accepted muons.

Figure 11 shows the performance of the front end as a function of taper length for target fields of 15 and $20 \mathrm{~T}$, and for final fields of 1.5 and 2.5 T. For all cases, when the taper length gets very short (below about $4 \mathrm{~m}$ ), the performance falls rapidly, because the taper is no longer sufficiently adiabatic. As the taper length increases beyond about $5 \mathrm{~m}$, the increase in longitudinal phase volume from the longer taper (Figs. 7-10) reduces the capture efficiency much more quickly than the better transverse emittance 


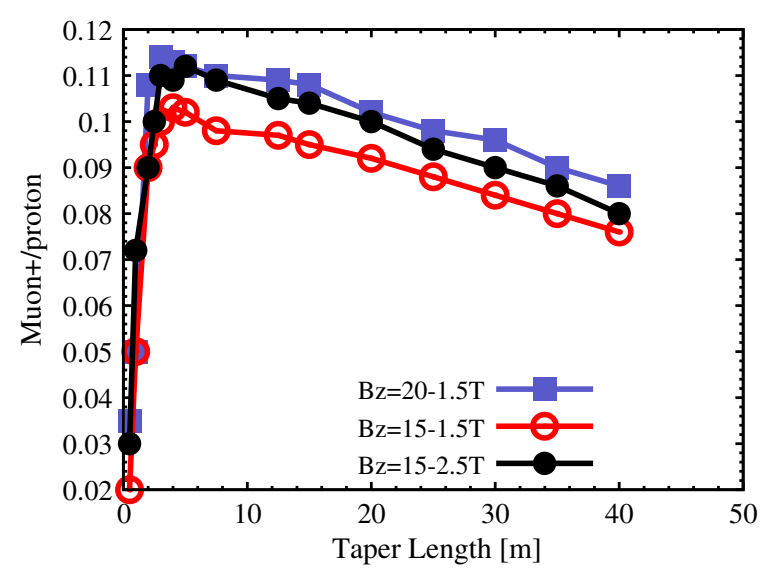

FIG. 11. Performance of the front end, in terms of accepted positive muons per $8 \mathrm{GeV}$ proton on target, as a function of taper length. Curves are shown for target fields of 15 and $20 \mathrm{~T}$, and final fields of $1.5,2.0$, and $2.5 \mathrm{~T}$.

preservation (Fig. 10) can improve it. We thus see a reduced performance for longer tapers. Higher fields at the target improve the performance since more particles are captured near the target (Fig. 5).

Increasing the final field increases the front end performance, as can be seen in Fig. 11. Figure 12 shows the performance with a $20 \mathrm{~T}$ target field and a $5 \mathrm{~m}$ taper length as a function of the final field. Performance increases by about $20 \%$ for every $1 \mathrm{~T}$ increase in the final field, and appears to be continuing to increase even with final fields as high as $3.5 \mathrm{~T}$. The improved performance of the front end with higher final fields can be explained by the fact that the acceptance of the constant field channel increases with the focusing field strength. The acceptance volume of an adiabatic taper is proportional to $q B a^{2}$, where $a$ is the physical aperture of the constant focusing channel. The strength of the end field determines the maximum confined transverse momentum of the captured beam. Increasing this field will increase the number of confined particles. For

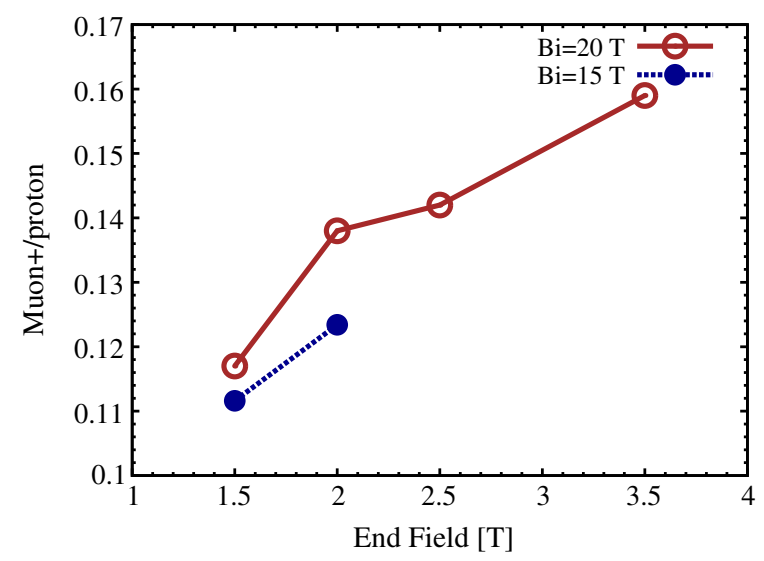

FIG. 12. Accepted positive muons per $8 \mathrm{GeV}$ proton on target as a function of the final field. The target field is 20 and $15 \mathrm{~T}$, and the taper length in both cases is $5 \mathrm{~m}$.



FIG. 13. Schematic showing a set of realistic superconducting solenoids giving a good approximation to a field profile having a $20 \mathrm{~T}$ target field, $2.5 \mathrm{~T}$ final field, and $5 \mathrm{~m}$ taper length. Rectangles are solenoids. The solenoids near $z=0$ with radius less than $1 \mathrm{~m}$ are resistive, the rest are superconducting. A curve giving the solenoid field on axis is also shown.

constant focusing solenoid field $B$, particles with transverse momentum $P_{t}<q B a / 2$ will be confined. Global optimization of the phases and frequencies of the rf cavities in the buncher and rotator in addition to the matching section to the ionization cooling channel and decay channel was essential to optimize the performance of the front end after increasing the end field [26].

Figure 13 shows a magnet configuration that will produce a field profile close to the theoretical profile we describe, with a target field of $20 \mathrm{~T}$, a final field of $2.5 \mathrm{~T}$, and a taper length of $5 \mathrm{~m}$, which from Fig. 11 is close to an optimal configuration. To produce the high field near the target, water-cooled resistive magnets are used near the target, and superconducting solenoids are used at larger radii. Sufficient space is included inside the superconducting magnets to shield them from the particles produced in the target by a $4 \mathrm{MW}$ proton beam. Shorter tapers are in fact simpler and less costly to construct than longer ones because fewer high field solenoids are needed.

The constant field after the end of the taper is approximated with a discrete set of solenoids, as shown in the right part of Fig. 13. The field from the actual solenoids will introduce stop bands and nonlinearities that will reduce transmission by about $2 \%$ compared to a constant solenoid field.

\section{EFFECT OF PROTON BUNCH LENGTH AND THE APERTURE SIZE ON THE PERFORMANCE}

We have shown that increasing the time spread in the pions and muons reduces the capture efficiency of the front end. Up to now all computations have been done with no time spread in the proton bunch that hits the target and produces these particles. An increase in the time spread in 


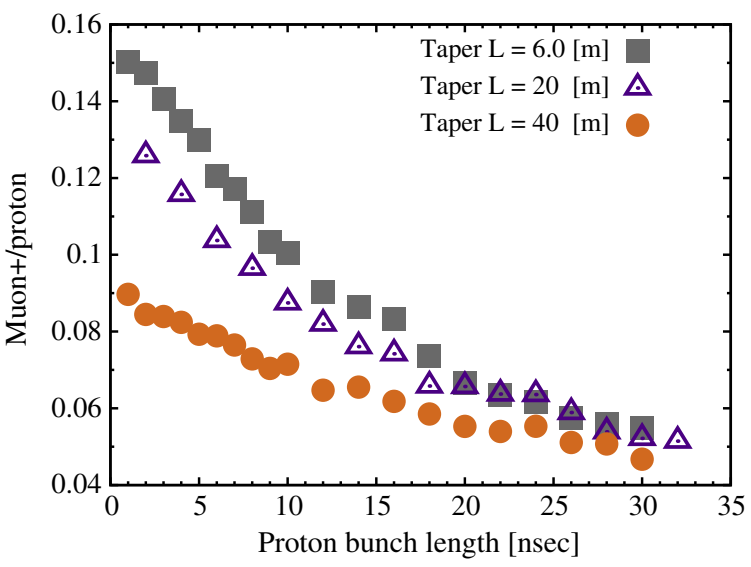

FIG. 14. Accepted positive muons as a function of the proton bunch length for three different taper lengths. The target field is $20 \mathrm{~T}$, and the final field is $3.5 \mathrm{~T}$.

the proton bunch would also be expected to reduce the capture efficiency of the front end [27]. For longer proton bunches, the time spread arising from a longer taper will make a smaller relative contribution to the capture efficiency, and thus for a sufficiently long proton bunch, a short taper will lose its advantage.

Figure 14 shows the performance of the front end as a function of the proton bunch length for three different taper lengths. Performance is reduced by approximately $3 \%$ for each $1 \mathrm{~ns}$ increase in proton bunch length. Once the proton bunch length reaches about $20 \mathrm{~ns}$, there is no significant performance difference between a $6 \mathrm{~m}$ taper and a $20 \mathrm{~m}$ taper, though a $40 \mathrm{~m}$ taper still has worse performance. Neutrino factory and muon collider designs specify $2-3 \mathrm{~ns}$ proton bunch lengths. Thus the designs are well within the region where there is an advantage in having a short taper. The advantage of short taper holds for short bunch lengths with 20 or $15 \mathrm{~T}$ peak field and various end fields as well.

\section{SUMMARY}

We have examined how the performance of a solenoid based capture channel is determined by the longitudinal profile of that field for a beam with large initial energy spread and transverse momentum spread. We have shown that the capture efficiency and transverse emittance are improved near the target by using high fields at the target. We have shown that reducing the length over which the field tapers down to smaller fields improves longitudinal phase space density, and therefore capture efficiency, until the taper length becomes so short as to create significant transverse emittance growth. We showed that the reduction in performance with increased taper length arises from particles with larger transverse amplitudes taking longer times to travel a given longitudinal distance. We found that up to reasonably high final taper field values, increasing the final taper field improved the capture efficiency of our system. Finally, we demonstrated that capture efficiency is reduced for a longer proton bunch length at the target, and that for bunch lengths which give a reasonable performance, there is still a preference for relatively short taper lengths.

These studies were applied to the design of a capture system for muons for a neutrino factory or muon collider, and will lead to improved performance for these machines, but the underlying mechanisms can be applied to other systems. In particular, the large acceptance of high solenoid fields will hold for pointlike particle sources, and shorter tapers will reduce longitudinal emittance growth for sources producing particles with large transverse emittances.

\section{ACKNOWLEDGMENTS}

We would like to thank H. Kirk, K. McDonald, R. Palmer, R. Ryne, D. Stratakis, and J. Qiang for useful discussions on the topics covered in this paper. S. Reyes of CERN helped us greatly by retrieving some important historical papers from the CERN archives scanning into the CERN Document Server. This effort was supported by the Office of High Energy Physics of the U.S. Department of Energy under Contract No. DE-AC02-98CH10886.

\section{APPENDIX: EMITTANCE AND ACCEPTANCE}

The precise definition of emittance and acceptance varies widely in the literature, so we will attempt, in this Appendix, to be specific about what we are using. Our values for emittance and acceptance are in most cases obtained using the program ECALC9F. The algorithms used therein are described in [17], and these are the definitive specifications of what we compute. However, we think it may be helpful to give some description of the basic ideas here.

By emittance we mean the rms or statistical definition of emittance. Our usage is consistent with that of Sacherer [21], though we will consider all six phase space dimensions. The starting point for computing the emittance is always the covariance matrix, whose elements are

$$
\Sigma_{\xi_{i}, \xi_{j}}=\left\langle\xi_{i} \xi_{j}\right\rangle-\left\langle\xi_{i}\right\rangle\left\langle\xi_{j}\right\rangle
$$

The $\xi_{i}$ are the phase space variables, and angle brackets are an average of the quantity in question over the distribution. The emittances are computed from the covariance matrix by taking the magnitudes of the eigenvalues of $\Sigma J$ (the eigenvalues in $2 n$ phase space dimensions come in $n$ imaginary complex conjugate pairs), where $J$ is the symplectic metric, which is a matrix with the $2 \times 2$ matrices

$$
\left[\begin{array}{cc}
0 & 1 \\
-1 & 0
\end{array}\right]
$$

on the diagonal and zeros elsewhere [28]. This definition is consistent with Sacherer's [21] in two phase space dimensions. 
Computing emittances in this fashion only has a good correspondence to what one expects (a phase space area occupied by the "useful" portion of the beam) when the distribution is reasonably ellipsoidal. A distribution which is highly nonellipsoidal, or which has some sort of tail or halo on it, can result in an emittance value which is determined by these other features. To avoid problems from halos and tails, ECALC9F uses an iterative process to remove particles in the tails. The basic idea is to first find a covariance matrix, then using the eigenvectors of that covariance matrix times $J$, transform the phase space such that the ellipsoid that the covariance matrix describes becomes a new ellipsoid where the projection of that ellipsoid into the each three "eigenplanes" corresponding to the matched eigenvalue pairs becomes a circle. One then removes all particles whose radius in any one of those circles lies outside of some multiple of the square root of the corresponding emittance. The process is repeated until no further particles are removed. Particles removed by this process are assumed lost to the system's transmission. See [17] for the precise details.

Throughout the paper we use normalized emittances. For transverse variables, it is conventional to define emittances based on phase space variables where the transverse momenta are scaled by the total momentum [29] or the longitudinal momentum (this is the historical convention $[20,21,30,31])$. Emittances defined this way will be reduced as the beam accelerates. To produce emittances that do not change as the beam accelerates, one defines normalized emittances to be these conventional emittances multiplied by $p /(m c)$, where $p$ is the total momentum, $m$ is the mass of the particle in question, and $c$ is the speed of light [31,32]. Emittances as described above would have units of eVs. To convert these emittances to the more conventional unit of $\mathrm{m}$, we divide by the constant $m c$.

Longitudinal and transverse emittances are projections, in that they are based on a submatrix of $\Sigma$. The longitudinal emittance is based on the time-energy sub-block. The transverse emittance is the square root of the product of the emittances for the $4 \times 4$ sub-block involving the transverse positions and momenta. See [17] for the precise algorithm.

We also discuss the number of particles falling within given transverse and longitudinal acceptances. In two phase space dimensions, we define the acceptance to be the area, divided by $\pi$, of an ellipse in phase space inside of which all particles will be transmitted [30]. We do not define a lattice that matches our distribution into some given machine with a particular acceptance. Instead, assuming such matching can be designed, we use the shape of the ellipses defined by the eigenvectors of the covariance matrix (see discussion above) to define the proper orientation and aspect ratio of the ellipses. The basic procedure is to transform the particles using the eigenvectors from the last iteration of the tail removal process described above, and count all particles with a squared radius in each eigenplane less than the corresponding acceptance (transverse or longitudinal). In the transverse we actually use the sum of the squares of the radii in the two transverse eigenplanes. The precise algorithm used is in [17].

Finally, all of our computations will be performed using kinetic momenta. This is in accord with the conventional definitions of emittance, but presents a difficulty in the presence of solenoid fields, where the kinetic and canonical momenta can differ significantly. It is the emittances in canonical variables that are preserved by linear symplectic transformations. However, we avoid the difficulty by our definition of transverse emittance: since the transformation from kinetic to canonical variables has determinant 1 , the product of the transverse emittances will be the same whether one is using kinetic or canonical momenta.

[1] M. Alsharoa et al., Phys. Rev. ST Accel. Beams 6, 081001 (2003).

[2] C. M. Ankenbrandt et al., Phys. Rev. ST Accel. Beams 2, 081001 (1999).

[3] C. Ankenbrandt, S. A. Bogacz, A. Bross, S. Geer, C. Johnstone, D. Neuffer, and M. Popovic, Phys. Rev. ST Accel. Beams 12, 070101 (2009).

[4] R. J. Weggel, N. Souchlas, H. K. Sayed, J. S. Berg, H. G. Kirk, X. Ding, V. B. Graves, and K. T. McDonald, in Proceedings of IPAC2013, Shanghai, China (JACoW, Shanghai, China, 2013), pp. 1514-1516, http://www .jacow.org/.

[5] C. T. Rogers, D. Stratakis, G. Prior, S. Gilardoni, D. Neuffer, P. Snopok, A. Alekou, and J. Pasternak, Phys. Rev. ST Accel. Beams 16, 040104 (2013).

[6] R. H. Helm, in The Stanford Two-Mile Accelerator, edited by R. B. Neal (W.A. Benjamin, New York, 1968).

[7] R. Palmer, AIP Conf. Proc. 887, 35 (1996).

[8] K. Paul and C. Johnstone, AIP Conf. Proc. 721, 329 (2004).

[9] C. Albright et al., Reports Nos. BNL-72369-2004, FNALTM-2259, and LBNL-55478, part of the American Physical Society Joint Study on the Future of Neutrino Physics: The Neutrino Matrix, http://www.aps.org/policy/reports/ multidivisional/neutrino/.

[10] J. S. Berg, S. A. Bogacz, S. Caspi, J. Cobb, R. C. Fernow, J. C. Gallardo, S. Kahn, H. Kirk, D. Neuffer, R. Palmer, K. Paul, H. Witte, and M. Zisman, Phys. Rev. ST Accel. Beams 9, 011001 (2006).

[11] K. Paul and C. Johnstone, Report No. NFMCC-doc-289, 2004, http://nfmcc-docdb.fnal.gov/.

[12] J. J. Back, C. Densham, R. Edgecock, and G. Prior, Phys. Rev. ST Accel. Beams 16, 021001 (2013).

[13] J. Berg, K. Long, and J. Pozimski, Proc. Sci., C1205201 (2012) 244.

[14] J. Strait, N. V. Mokhov, and S. I. Striganov, Phys. Rev. ST Accel. Beams 13, 111001 (2010).

[15] N. Mokhov and S. Striganov, AIP Conf. Proc. 896, 50 (2007). 
[16] D. Neuffer, M. Martini, G. Prior, C. Rogers, and C. Yoshikawa, in Proceedings of the International Particle Accelerator Conference, Kyoto, Japan (ICR, Kyoto, 2010), WEPE068.

[17] R.C. Fernow, Physics Analysis Performed by ECALC9 (Brookhaven National Laboratory, Upton, New York, 2003).

[18] R. C. Fernow, in Proceedings of 2005 Particle Accelerator Conference, Knoxville, Tennessee (IEEE, New York, 2005), pp. 2651-2653.

[19] E. D. Courant, R. D. Ruth, and W. T. Weng, AIP Conf. Proc. 127, 294 (1985).

[20] R. Hagedorn, M. G. N. Hine, and A. Schoch, CERN Symposium on High Energy Accelerators and Pion Physics, edited by E. Regenstreif (CERN, Geneva, Switzerland, 1956), Vol. 1, pp. 237-253, their $a$ is the action.

[21] F. J. Sacherer, IEEE Trans. Nucl. Sci. 18, 1105 (1971).

[22] A. J. Lichtenberg, Rev. Sci. Instrum. 34, 1196 (1963).

[23] V. E. Cosslett, Introduction to Electron Optics (Oxford, Oxford, 1946), Chap. 4, p. 106, Eqs. IV.45 and IV.46.

[24] J. R. M. Vaughan, IEEE Trans. Electron Devices 19, 144 (1972), Eqs. (4) and (5).
[25] J. S. Berg, Nucl. Instrum. Methods Phys. Res., Sect. A 570, 15 (2007).

[26] H. Sayed, J. Berg, H. Kirk, R. Palmer, D. Stratakis, K. McDonald, D. Neuffer, J. Qiang, and R. Ryne, in Proceedings of the North American Particle Accelerator Conference NAPAC2013, Pasadena, CA, 2013.

[27] J. C. Gallardo, Technical Report, Brookhaven National Laboratory, 2006.

[28] F. Neri and G. Rangarajan, Phys. Rev. Lett. 64, 1073 (1990).

[29] R. D. Ruth, in Nonlinear Dynamics Aspects of Particle Accelerators, Lecture Notes in Physics Vol. 247, edited by J. M. Jowett, M. Month, and S. Turner (Springer, New York, 1986), pp. 37-63.

[30] H. G. Hereward, K. Johnsen, and P. Lapostolle, in CERN Symposium on High Energy Accelerators and Pion Physics (Ref. [20]), pp. 179-191, acceptance is defined in Sec. 2.3 of Part II

[31] A. van Steenbergen, IEEE Trans. Nucl. Sci. 12, 746 (1965).

[32] A. van Steenbergen, Technical Report No. AADD-29, 1964, http://cds.cern.ch/record/1044855/. 